\title{
Erratum to: The phylogeny of Arthoniales (Pezizomycotina) inferred from nucLSU and $R P B 2$ sequences
}

\author{
Damien Ertz • Anders Tehler
}

Published online: 10 May 2011

(C) Kevin D. Hyde 2011

\section{Erratum to: Fungal Diversity}

$$
\text { DOI 10.1007/s13225-010-0080-y }
$$

The original publication contains the following error (7th page, bottom of left column):

'Dendrographa latebrarum (Egea \& Torrente)' should be 'Dendrographa latebrarum (Ach.)'

The online version of the original article can be found at http://dx.doi. org/10.1007/s13225-010-0080-y.

D. Ertz $(\bowtie)$

Department Bryophytes-Thallophytes,

National Botanic Garden of Belgium,

Domein van Bouchout,

1860 Meise, Belgium

e-mail: damien.ertz@br.fgov.be

\section{A. Tehler}

Enheten för kryptogambotanik, Naturhistoriska riksmuseet,

Box 50007, 10405 Stockholm, Sweden

e-mail: Anders.Tehler@nrm.se 